\title{
Single-trial ERP estimation based on spatio-temporal filtering
}

\author{
Ruijiang Li, Jose C. Principe, Margaret Bradley, Vera Ferrari
}

\begin{abstract}
This paper introduces a new approach to the problem of single-trial event-related potentials (ERP) estimation in EEG data with very low signal-to-noise (SNR) ratio. The method relies on modeling of the ERP component descriptors to be estimated and utilizes a spatial filter to constrain the output in the temporal domain. Results on cognitive EEG data with very low SNR show that our method successfully extracts the P300 component while other traditional methods fail. Some possible improvements on the present method are also discussed.
\end{abstract}

\section{INTRODUCTION}

$\mathrm{T}$ raditional event-related potentials (ERP) analysis methods such as trial-averaging usually only considers the time course of a single channel in Electroencephalography (EEG). Today, high-density EEG can simultaneously record scalp potentials in up to 256 electrodes. This increased number of sensors and thus increased spatial resolution has created a need for methods that can analyze the time series of multiple channels simultaneously and will lead to an enhanced signal-to-noise ratio (SNR).

Recently, various signal processing methods have been proposed for EEG analysis that linearly combine the time series in multiple channels to generate a representation of the observed data that is easier to interpret [1]-[8]. Specifically, denote the multidimensional EEG data by a $D \times T$ matrix $\mathbf{X}$, where $D$ is the number of channels and $T$ is the number of samples in time. A weight vector $\mathbf{W}$ can be selected to generate a time series (sometimes called a "component") denoted by the one-dimensional vector $\mathbf{y}=\mathbf{w}^{T} \mathbf{X}$.

This linear projection combines the information from all available sensors into a single channel whose time course can then be analyzed with conventional methods, such as, trial averaging, temporal filtering, frequency analysis, etc. When applied to the EEG data in multiple channels, the weight vector $\mathbf{W}$ can reduce interference from other neural sources and may provide a better estimate of the underlying neural activity than the EEG measurements in a single sensor. The weight vector $\mathbf{w}$, in this sense, may be called a

Manuscript received January 25, 2007. This work was jointly supported by Graduate Alumni Fellowship from University of Florida and the National Institute of Mental Health (NIMH) grant P50 MH072850-01.

R. Li and J. Principe are with the Computational NeuroEngineering Lab, Electrical and Computer Engineering Department, University of Florida, Gainesville, FL 32611 USA. corresponding author: R. Li, phone: 352-392-2682; fax: 352-392-0044; (e-mail: ruijiang@cnel.ufl.edu, principe@cnel.ufl.edu)

M. Bradley and V. Ferrari are with NIMH Center for the Study of Emotion and Attention, University of Florida, Gainesville, FL 32611 USA. (email: bradley@ufl.edu, ferrari@biocfarm.unibo.it) "spatial filter". Because of the improved SNR with the application of the spatial filter, ERP estimation may be achieved on a single-trial basis, instead of using the traditional methods such as trial-averaging.

The selection of $\mathbf{w}$ is based on some constraints or desired characteristics of the component $\mathbf{y}$. Different constraints lead to different methods of extracting the components. Loosely speaking, maximum power leads to PCA [1]; maximum difference leads to linear discriminant analysis [6]; statistical independence leads to ICA [3]. One of the difficulties for these methods is that they generally do not work for cases where the desired component has very small power in EEG data.

In this study, we propose a spatio-temporal filtering method that will constrain through the spatial filter $\mathbf{w}$ the extracted ERP component to have minimal distance in the temporal domain from a presumed ERP component. The model allows for amplitude and latency variability in the actual component. Note that we do not constrain the entire ERPs, but instead a single ERP component.

The organization of the paper is as follows. Section 2 introduces a generally accepted generative EEG model, which serves as the foundation for our method. Section 3 presents the details of the spatio-temporal filtering approach and constitutes the main body of the paper. Some results on single-trial ERP estimation from clinical EEG data are presented in section 4 . Section 5 discusses some implications and possible improvements of the method.

\section{LINEAR GENERATIVE EEG MODEL}

We start with the "neuronal generator" assumption of EEG data, i.e., neuron populations in cortical and subcortical brain tissues act as current sources [9], [10]. Within the EEG frequency range, brain tissues can be assumed to be primarily a resistive medium governed by Ohm's law [11]. Thus, in EEG data, the electrical potentials collected at each sensor as a result of volume conduction, is basically a linear combination of neural current sources (and non-neural artifacts). The linear generative model for EEG data can be written in matrix form:

$$
\mathbf{X}=\mathbf{a} \cdot \mathbf{s}^{T}+\sum_{i=1}^{N} \mathbf{b}_{i} \mathbf{n}_{i}^{T}
$$

where, $\mathbf{X}$ is a $D \times T$ matrix representing the single-trial EEG data, with $D$ channels and $T$ samples. $\mathbf{S}$ is the time course of ERP component to be extracted, $\mathbf{n}_{i}$ denotes noise in general. The distinction between "source" and "noise" is somewhat arbitrary, e.g., when P300 is the signal of interest, N100 will become noise in the model. Strictly speaking, the number of the neural sources is 
necessarily larger than the number of sensors. It is usually assumed that the numbers of sources and sensors are equal for the purpose of convenience. Here, We have $N \gg D$.

The EEG model in (1) can be rewritten as follows:

$$
\mathbf{X}=\sigma_{s} \mathbf{a}_{o} \cdot \mathbf{s}_{o}{ }^{T}+\sum_{i=1}^{N} \sigma_{i} \mathbf{b}_{i} \mathbf{n}_{i}{ }^{T}
$$

where, $\mathbf{a}_{o}, \mathbf{s}_{o}, \mathbf{b}_{i}^{\prime}, \mathbf{n}_{i}^{\prime}$ are the normalized versions (unit norm) of their counterparts in (1) and $\sigma_{i}>\sigma_{i+1}$. The scalars $\sigma_{s}, \sigma_{i}$ can be seen as the overall contributions of the sources to the single-trial EEG data.

The vectors $\mathbf{a}_{o}, \mathbf{b}_{i}^{\prime}$, represent the projection of the corresponding signal to each sensor at the scalp and are called the forward model associated with the signal. They are generally unknown and depend on the orientation of the current source as well as the conductivity distribution of the underlying brain tissues, skull, skin and electrodes [12]. For a meaningful ERP component, it must have a stable scalp projection $\mathbf{a}_{o}$. Thus, we may assume that $\mathbf{a}_{o}$ is fixed for all trials. We also assume that the waveform of a particular ERP component $\mathbf{s}_{o}$ (dimensionless) remains the same for all trials, although its amplitude $\sigma_{s}$ may change across trials.

\section{SPATIO-TEMPORAL FILTERING METHOD}

In the ERP research community, it is conventional to analyze the complete ERP as a superposition of several psychologically meaningful components. The distinction and definition of these ERP components are usually based on their polarity and latency. Fabiani, Gratton, Karis and Donchin in [13] gave an excellent survey of the observational definitions of ERP components and discussed their advantages and disadvantages in the context of the P300.

We assume a Gaussian waveform for a particular ERP component with a variable peak amplitude and latency. Lange et al showed that if the firing instants of many synchronized neurons are assumed to be Gaussian, the resulting ERP component will approximately have a Gaussian shape [14]. We note that the extracted ERP component need not be a Gaussian waveform. It is also worth mentioning that although in reality the actual ERP component may not be a Gaussian waveform, moderate deviations would not distort significantly the estimations of peak amplitude and latency, but might affect the tails of overlapping components. This is not crucial, however, since neurophysiologists are primarily concerned with peak parameters (latency and amplitude), rather than the exact waveform.

Next, we claim without proof the following lemma. Basically it is a consequence of the linear generative EEG model introduced in the previous section.

Lemma: There exists a spatial filter $\mathbf{w}$, such that the SNR in the extracted ERP component can be greatly enhanced when it is applied to the single-trial EEG data $\mathbf{X}$ if,

(1), $\sigma_{s} \gg \sigma_{D}$

(2), there is no $i$ such that $\operatorname{angle}\left(\mathbf{a}, \mathbf{b}_{i}\right) \rightarrow 0$.

\section{A. Finding the peak latency for ERP component}

Obviously, the optimal spatial filter $\mathbf{w}$ depends on which ERP component is to be extracted, and thus is a function of the unknown peak latency $l$. The search for $\mathbf{w}(l)$ could be realized by minimizing some distance measure between $\mathbf{w}(l)^{T} \cdot \mathbf{X}$ and the assumed waveform $\mathbf{s}_{o}(l)$ for the particular ERP component. We propose the following cost function based on second-order statistics (SOS):

$$
\min J(l)=\left\|\mathbf{w}(l)^{T} \cdot \mathbf{X}-\mathbf{s}_{o}(l)^{T}\right\|_{2}^{2}
$$

The optimal solution for $\mathbf{w}(l)$ is given by:

$$
\mathbf{w}(l)=\left(\mathbf{X X}^{T}\right)^{-1} \cdot \mathbf{X} \cdot \mathbf{s}_{o}(l)
$$

In practice, since $\mathbf{X} \mathbf{X}^{T}$ is almost always rank deficient, the above solution is replaced by:

$$
\mathbf{w}(l)=\left(\mathbf{X X}^{T}+\lambda \mathbf{I}\right)^{-1} \cdot \mathbf{X} \cdot \mathbf{s}_{o}(l)
$$

where, $\lambda$ is the regularization parameter.

The peak latency of the ERP component can be set as $l$ where the local minimum of $J(l)$ occurs within the range of meaningful peak latencies for that component.

\section{B. Finding the scalp projection and amplitude for ERP component}

In order to estimate the unknown scalp projection and amplitude of the ERP component, we make a further assumption: the ERP component is uncorrelated with all the other "noises". Although it is not fully justified and sometimes subject to interpretation, the uncorrelatedness assumption is generally accepted [1], [3], [4], [12]. Some methods such as ICA admit an even stronger assumption of independence among the outputs [3], [4].

In the following notations, we make the index for trial number $k$ explicit. Denote the ERP component for $k$ th trial by (the peak latency of the ERP component depends on the trial number):

$$
\mathbf{s}_{k}\left(l_{k}\right)=\sigma_{s_{k}} \mathbf{s}_{o}\left(l_{k}\right)
$$

We can absorb the scalar $\sigma_{s_{k}}$ into a variable scalp projection:

$$
\mathbf{a}_{k}=\sigma_{s_{k}} \mathbf{a}_{o}
$$

Multiply $\mathbf{s}_{o}(l)$ on both sides of (2), assuming uncorrelatedness of the ERP component and the noise, we get,

$$
\hat{\mathbf{a}}_{k}=\frac{\mathbf{X}_{k} \mathbf{s}_{o}\left(l_{k}\right)}{\mathbf{s}_{o}^{T}\left(l_{k}\right) \mathbf{s}_{o}\left(l_{k}\right)}
$$


(Note that $\hat{\mathbf{a}}_{k}$ has the same dimension as $\mathbf{X}_{k}$ i.e., $m V$ ). Take the normalized version, and we have,

$$
\hat{\mathbf{a}}_{k}^{\prime}=\frac{\hat{\mathbf{a}}_{k}}{\sqrt{\hat{\mathbf{a}}_{k}{ }^{T} \hat{\mathbf{a}}_{k}}}
$$

Ideally, the normalized $\hat{\mathbf{a}}_{k}^{\prime}$ should be the same as the dimensionless scalp projection $\mathbf{a}_{o}$. However, in low-SNR EEG data, the above estimation in (9) is very poor, due to the interference from larger noises in finite-sample data. An obvious improvement is to take the average across all trials. And we obtain the following estimate for $\mathbf{a}_{o}$ :

$$
\hat{\mathbf{a}}_{o}=\frac{1}{K} \sum_{k=1}^{K} \hat{\mathbf{a}}_{k}^{\prime}
$$

Notice that (10) is a ML estimator for $\mathbf{a}_{o}$ assuming Gaussian statistics. We also point out that (10) is different from summing up directly (8) for all trials since the peak latency parameter is involved and it changes from trial to trial.

Having found the estimates for $\mathbf{a}_{o}$ and $\mathbf{a}_{k}$, the only unknown parameter is the amplitude $\sigma_{s_{k}}$. Replacing their respective estimates in (8), we can find $\sigma_{s_{k}}$ using again SOS criterion:

$$
\min J=\left\|\hat{\mathbf{a}}_{k}-\sigma_{s_{k}} \hat{\mathbf{a}}_{o}\right\|_{2}^{2}
$$

Simple calculation leads to the following estimate for the amplitude:

$$
\hat{\sigma}_{s_{k}}=\hat{\mathbf{a}}_{o}^{T} \cdot \hat{\mathbf{a}}_{k}
$$

These estimates for scalp projection, peak latencies and amplitudes of ERP components can then be used to analyze their psychological relevance on a single-trial basis.

\section{RESULTS}

The EEG data were recorded from one subject during a passive picture-viewing experiment, which consists of 12 alternating phases: the habituation phase and novel phase. Each phase has 30 trials. During the 30 trials of the habituation phase, the same picture was repeatedly presented 30 times. During the novel phase, the 30 pictures are all different. Each trial lasts $1600 \mathrm{~ms}$, and there is $600 \mathrm{~ms}$ pre-stimulus, and $1000 \mathrm{~ms}$ post-stimulus.

The scalp electrodes were placed according to 128-channel Geodesic Sensor Nets standards. All 128 channels were referred to $\mathrm{Cz}$ and were digitally sampled for analysis at $250 \mathrm{~Hz}$. A bandpass filter between $0.1 \mathrm{~Hz}$ and $40 \mathrm{~Hz}$ was applied to all channels, which were then converted to average reference. Common artifacts such as cardiac artifacts and eye movement and blinking artifacts were removed from the EEG data prior to analysis.

Here, we are primarily interested in the estimation of the P300 component. We assume a Gaussian shape for the P300 component with variable peak latency and amplitude. For this paper, the only shape parameter that needs to be determined is the variance (or spread) of the Gaussian

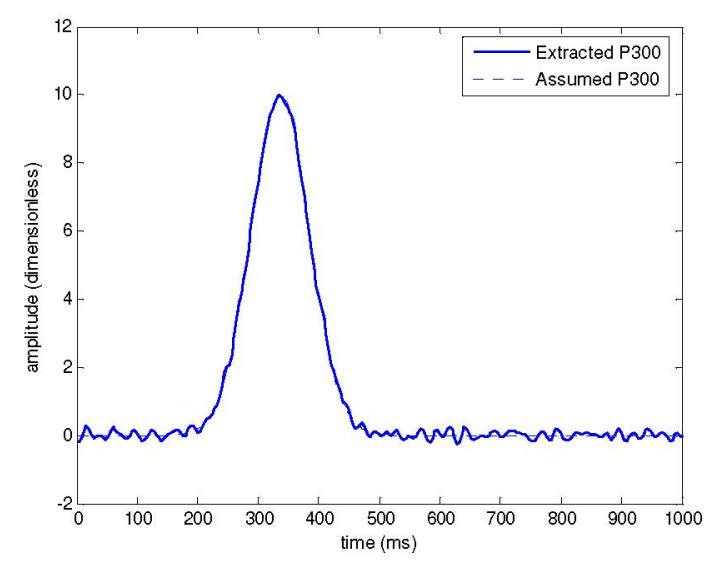

Fig. 1. a typical extracted P300 and the presumed P300 component

waveform. The selection of the spread parameter is not optimized at this point. We instead used several spread parameters within the range of $40 \mathrm{~ms}$ and $60 \mathrm{~ms}$ and the results were found to be similar.

Fig. 1 shows a typical extracted P300 component. Also shown is the presumed P300 component with a spread of $48 \mathrm{~ms}$. Note that the extracted P300 component is dimensionless. It has physical meaning only when it is multiplied by the scalar $\sigma_{s_{k}}$. The extracted P300 components are very similar for all trials.

The P300 peak latency is set as the first local minimum after $300 \mathrm{~ms}$ of the stimulus onset. Fig. 2 shows a histogram of estimated P300 peak latencies, which is consistent with the fact that P300 normally occurs between $300 \mathrm{~ms}-800 \mathrm{~ms}$ after stimulus. Average peak latency of P300 for single trials is around 355 ms post-stimulus.

Fig. 3 shows the estimated scalp projection of P300 from all trials. We notice that instead of having a parietal maximum for a classical P300, the scalp projection of the estimated P300 component shifts its maximal projection to the occipital areas. This may arise from the poor ML estimator in (10). On the other hand, it might reflect the subject variability or the specificity of the experimental design. The estimation is fairly stable. If we estimate the scalp projection from 30 trials ( 1 phase), the correlation coefficients among the 12 phases are largely between 0.7 and 1 .

We briefly mention the results on the estimated P300 amplitude. Basically there was a $13 \%$ drop in P300 peak amplitude from the first 10 trials to the last 10 trials for the 6 habituation phases, while there was no obvious change for the novel phases. This confirmed the habituation effect when the same stimulus is repeatedly presented to the subject.

\section{DISCUSSIONS}

The clinical EEG data we have used is one of the cases where the signal of interest (P300) has very small power 
relative to noise (background $\mathrm{EEG).} \mathrm{In} \mathrm{this} \mathrm{case,} \mathrm{methods}$ such as PCA and ICA will usually fail. Our method relies

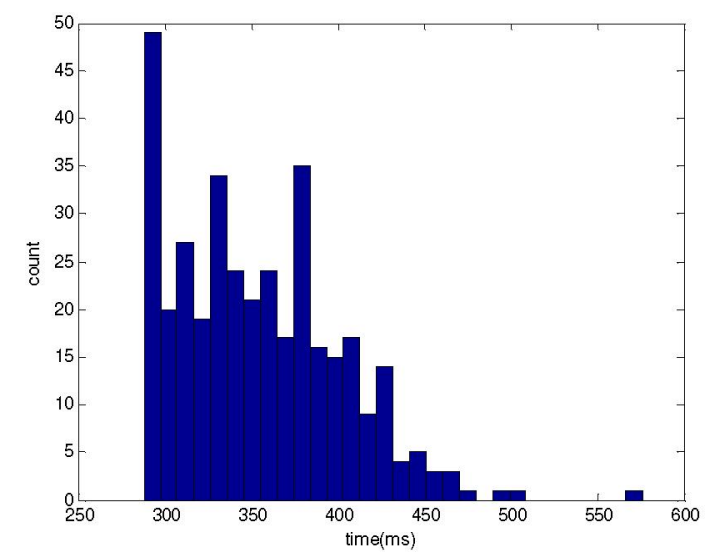

Fig. 2. histogram of the estimated P300 peak latencies

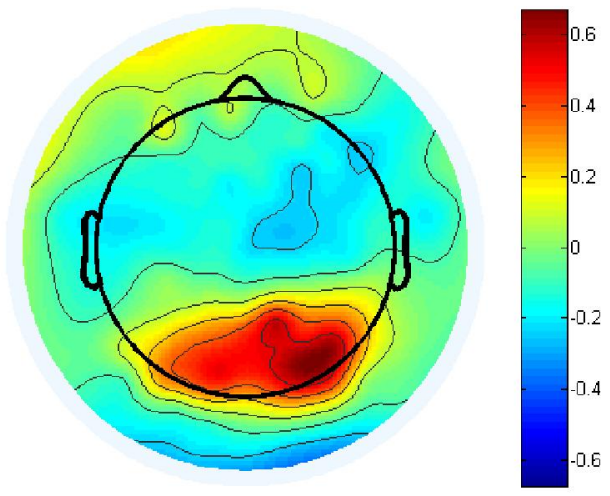

Fig. 3. estimated scalp projection for P300 from all trials

on modeling of the desired signal descriptors and is tailored to treat EEG data with very low SNR. It can be applied to cases where some a priori knowledge about the ERP component is known but is not suitable to discover "new" ERP component. We also point out that our method is not limited to estimate the P300 component. It is also able to estimate other well-known ERPs, such as N100, P200, provided that a suitable spread parameter is used.

We could have used mixtures of Guassians to represent the entire ERPs, but an easier approach is to estimate the ERP components one by one, as is done in this study. The exact shape of a ERP component depends on the underlying cognitive processing, which we have little knowledge about. For simplicity, we used a Gaussian shape. It is trivial to extend to other (perhaps more appropriate) shapes, e.g., Poisson, Laplacian or Gamma.

The assumption of the uncorrelatedness between the noise and ERP component is conceptually appealing, but temporal overlap of the ERP components (especially P300 and late positive complexes) may complicate the matter. Also, for finite-sample data, the ML estimation for $\mathbf{a}_{k}$ in (8) is poor because of the noisy nature of EEG data. An immediate improvement is to use regularization techniques with smoothness constraints to stabilize the solution. Other solutions based on robust statistics may also be useful.

\section{REFERENCES}

[1] Chapman, R., McCrary, J., EP component identification and measurement by principal components analysis. Brain Cogn. 27 (3), 288-310, 1995. (review, Erratum in: Brain Cogn. 28 (3) 342, 1995)

[2] Koles, Z., J.C., L., Soong, A., Spatio-temporal decomposition of the EEG: a general approach to the isolation and localization of sources. Electroencephalogr. Clin. Neurophysiol. 95 (4), 219-230 1995

[3] Makeig, S., Bell, A., Jung, T., Sejnowski, T., Independent component analysis of electroencephalographic data. Advances in Neural Information Processing Systems, vol. 8. MIT Press, 145-151, 1996

[4] Tang, A.C., Pearlmutter, B.A., Malaszenko, N.A., Phung, D.B., Reeb, B.C., Independent components of magnetoencephalography: localization. Neural Comput. 14 (8), 1827-1858, 2002

[5] Ramoser, H., Mueller-Gerking, J., Pfurtscheller, G., Optimal spatial filtering of single trial EEG during imagined hand movement. IEEE Trans. Rehabil. Eng. 8 (4), 441-446, 2000

[6] Parra, L.C., Alvino, C., Tang, A., Pearlmutter, B., Young, N., Osman, A., Sajda, P., Linear spatial integration for single-trial detection in encephalography. Neurolmage 17, 223-230, 2002

[7] Parra, L.C., Sajda, P., Blind source separation via generalized eigenvalue decomposition. J. Mach. Leam. Res. 4, 1261-1269, 2003

[8] Delorme, A., Makeig, S., EEGLAB: an open source toolbox for analysis of single-trial EEG dynamics including independent component analysis. J. Neurosci. Methods 134 (1), 9 -21, 2004

[9] Caspers, H., Speckmann, E.-J., \& Lehmenkühler, A. Electrogenesis of cortical DC potentials. In H. H. Kornhuber \& L. Deecke (Eds.), Progress in brain research: Vol. 54. Motivation, motor and sensory processes of the brain: Electrical potentials, behavior and clinical use 3-15. Amsterdam: Elsevier. 1980

[10] Sams, M., Alho, K., Näätänen, R. Short-term habituation and dishabituation of the mismatch negativity of the ERP. Psychophysiology, 21, 434-441. 1984

[11] Reilly, J., Applied Bioelectricity. Springer, 1992

[12] Parra, L., Spence, C., Gerson, A., Sajda, P., Recipes for the linear analysis of EEG. Neuroimage, 28, 326-341, 2005

[13] Fabiani, M., Gratton, G., Karis, D., \& Donchin, E. Definition, identification, and reliability of measurement of the P300 component of the event-related brain potential. In P. K. Ackles, J. R. Jennings, M. G. H. Coles (Eds.), Advances in psychophysiology Vol. 2, 1-78. Hillsdale, NJ: JAI Press, 1987

[14] Lange, D., Pratt, H., Inbar, G., Modeling and estimation of single evoked brain potential components, IEEE Trans. Biomed. Eng., 44, 791-799, 1997 